\title{
Enhancement of Coloured Image Quality Using Histogram Equalization Technique
}

\author{
Pooja \\ Department of Computer Science Engineering \\ Punjab Techanical University \\ Jalandhar, Punjab, India \\ Gurwinder Singh Jatana \\ Department of Computer Science Engineering \\ Punjab Techanical University \\ Jalandhar, Punjab, India
}

\begin{abstract}
Histogram Equalization (HE) is a simple and effective image enhancement technique. But, it tends to change the mean brightness of the image to the middle level of the permitted range, and hence is not a very suitable for consumer product. While preserving the original brightness is essential to avoid annoying artifacts. To preserve brightness and to enhance contrast of images, numerous methods are introduced, but many of them present unwanted artifacts such as intensity saturation, over-enhancement and noise amplification. In this paper we review the various methods for histogram equalization.
\end{abstract}

Keywords - Histogram equalization, Image, Brightness.

\section{INTRODUCTION}

The main objective of image enhancement is to procedure the image in order that the output image will be better than data image. So this technique enhances and improves the quality of the image.

Adaptive histogram equalization (AHE) [6] is a computer image processing technique utilized to improve contrast in images. It differs from ordinary histogram equalization in the respect that the adaptive method computes several histograms, every corresponding to a distinct section of the image, and uses them to redistribute the lightness values of the image. It is therefore suitable for improving the local contrast.

However, AHE has a tendency to over amplify noise in relatively homogeneous regions of an image. A variant of adaptive histogram equalization called contrast constrained adaptive histogram equalization (CLAHE) prevents this by limiting the amplification. AHE [5] is an extension to traditional Histogram Equalization technique. It enhances the contrast of images by transforming the values in the intensity image I. Unlike HISTEQ, it operates on small data regions (tiles), rather than the entire image. Every tile's contrast is enhanced, in order that the histogram of the output region approximately matches the specified histogram. The neighboring tiles are then combined using bilinear interpolation in order to eliminate artificially induced boundaries. The contrast, especially in homogeneous areas, can be constrained in order to avoid amplifying the noise which might be present in the image.

Ordinary histogram equalization uses the same transformation derived from the image histogram to transform all pixels. This works well when the distribution of pixel values is similar throughout the image. However, when the image contains regions that are altogether lighter or darker than most of the image, the contrast in those regions will not be sufficiently enhanced.

Adaptive histogram equalization (AHE) improves on this by transforming every pixel with a transformation function derived from a neighbourhood region. It was first developed for use in aircraft cockpit displays.

\section{LITERATURE REVIEW}

Muna Abdul Hussain Radhi et al.[9] studied the effect of the light distribution on the quality of captured images under different lightness conditions then enhancing the captured images using Histogram equalization method depending on the space YIQ depending on lightness component where use the reverse of this space to the basic RGB color space, then corrected using the mathematical model based on human vision system in this paper. The results were analyzed and compute the quality of the enhancement images by using two statistical criteria the mean and standard deviation. Histogram equalization technique gives a high quality for the enhanced images for different lightness conditions. 
Kim [8] proposed the first Mean based separation technique, Brightness Preserving Bi-Histogram Equalization (BBHE) to preserve the mean brightness of a given image while contrast is enhanced and it preserves the brightness of image at some extent and shown better result than HE.

Wang et al.[13] proposed Dualistic Sub-Image Histogram Equalization (DSIHE), but this method utilized median value instead of mean to separate the data histogram and shown better brightness preserving than BBHE and HE. DSIHE is the best procedureing technique to preserve the original image brightness and also enhance the image information effectively. BBHE and DSIHE are not much suitable for images requiring higher degree of brightness preservation to avoid annoying artifacts.

Chen et al.[3,4] proposed Minimum Mean Brightness Error Bi-Histogram Equalization (MMBEBHE) an extended method of BBHE and the separation based on threshold level, which would yield minimum Absolute Mean Brightness Error (AMBE). The ultimate goal behind this method is to allow maximum level of brightness preservation in Bi-Histogram Equalization to avoid unpleasant artifacts and unnatural enhancement due to excessive equalization, and also to formulate an efficient, recursive and integer-based solution to approximate the output mean as a function of threshold level. Simulated results from [8], MMBEBHE clearly indicates that it has preserved better brightness and yielded a more natural enhancement. BBHE and MMBEBHE have a better preservation and enhancement levels compared to HE and DSIHE. But, MMBEBHE shows poor brightness preservation and enhancement, where the images that require far more brightness preservation it fails to control the over enhancement of the image.

Sengee et al.[10] introduced the Histogram Equalization with Neighbourhood Metric (BHENM), which divide the large histogram bins that cause washout artifacts into sub-bins using neighbourhood metrics, and the histogram of the original image is separated into two sub-histograms based on the mean of the histogram. Then, sub-histograms are equalized independently using refined histogram equalization, which produce flatter histograms. The distinction neighbourhood metric is to sort pixels of equal intensity into different sub-bins, to improve image local contrast, and to separate the histogram into two sub-histograms and then equalize them independently to preserve the image brightness.

Zuo et al.[14] proposed the Range Constrained Bi-Histogram Equalization (RLBHE, which divides the data histogram into two independent sub-histograms by a threshold that minimizes the intra-class variance. This was carried out to effectively separate the objects from the background. This method achieves visually a more pleasing contrast enhancement while maintaining the data brightness and it is easy to implement in real-time procedureing.

Rajesh Garg et al.[5] discussed that various enhancement schemes are utilized for enhancing an image which includes gray scale manipulation, filtering and Histogram Equalization (HE). Histogram equalization is one of the well known image enhancement technique. It became a popular technique for contrast enhancement because this method is simple and effective. In the latter case, preserving the data brightness of the image is required to avoid the generation of non-existing artifacts in the output image. Although these methods preserve the data brightness on the output image with a significant contrast enhancement, they may produce images with do not look as natural as the data ones. The basic idea of HE method is to re-map the gray levels of an image. HE tends to introduce some annoying artifacts and unnatural enhancement. To overcome these drawbacks different brightness preserving techniques are utilized which are covered in the literature survey. Comparative analysis of different enhancement techniques will be carried out. This comparison will be done on the basis of subjective and objective parameters. Subjective parameters are visual quality and computation time and objective parameters are Peak signal to noise ratio (PSNR), Mean squared error (MSE), Normalized Absolute Error (NAE), Normalized Correlation, Error Color and Composite Peak Signal to Noise Ratio (CPSNR).

Sos S. Agaian et al. [1] introduced a new kind of "frequency domain" based signal/image enhancement algorithms (magnitude enrichment, log-magnitude reduction, iterative amplitude, and log-reduction zonal magnitude techniques) have been described and applied for detection and visualization on objects within an image. The new techniques are based on the so called "sequency" ordered orthogonal transforms, which include the well known fast orthogonal Fourier, Hartley, cosine, and Hadamard transforms, as well as new enhancement techniques. We have improved upon the current magnitude reduction techniques and developed an entirely novel method. The wide range of characteristics can be obtained from a single transform by varying enhancement parameters. A quantitative measure of signal/image enhancement was presented, which demonstrated the optimal method to automatically choose the best parameters and transform. The proposed technique is simple to design and implement, which makes them practical. A number of experimental results were given which illustrate the performance of these algorithms. The comparative analysis of transforms based image enhancement algorithms has been described, too. Lastly, the comparison of the Fourier transform and Walsh, cosine and Hartley transforms was given. They find that for a negligible trade off of accuracy, one can use the Walsh transform to achieve altogether higher performance enhancement. For our purposes, where 
speed is a major concern, the proposed method turns out to be a dramatic improvement over existing methods. They have also proposed the zonal transform based image enhancement algorithms.

Krishan Kant Lavania et al.[9] analyzed two image enhancement techniques based on spatial domain approach. Histogram Equalization generates a gray map which changes the histogram of an image and redistributing all pixels values to be as close as possible to a user specified preferred histogram. Histogram equalization allow for areas of lower local contrast to gain ahigher contrast. In Histogram design, data image has given, and then the target histogram is specified, image has procedureed in such a way that the histogram of the procedureed image will be close to the target image. The author evaluated and compared these two methods based on their EME values. The experimental results show that Histogram Specification is better than Histogram Equalization because the images are more cleared and the background is more detailed.

J. Alex Stark [11] et al. introduced a method for adaptive image contrast enhancement based on a generalization of histogram equalization (HE). HE is a useful technique for improving contrast of an image, but it is use for many purposes. However, severely different results can be obtained with relatively minor manipulations. A brief description of adaptive HE is set out, and this framework is utilized in a discussion of past suggestions for variations on HE. The primekey of this technique is a "cumulation function," which is utilized to produce a grey level mapping from the local histogram. By selecting alternative forms of cumulation function one can experience a wide variety of effects. A precise form is proposed. Through the variation of two or three parameters, the results can produce a dynamic range of contrast enhancement, at one severe leaving the image unaffected, on the other hand yielding full adaptive equalization.

Jinshan Tang et al. [12] described an image contrast enhancement algorithm that is based on a contrast measure defined in the DCT domain. The comparative analysis between the proposed algorithm and two existing algorithms has shown the merit of the contrast measure based approach.

Manpreet Kaur[7]et al. showed the comparative study of Histogram Equalization based methods. They showed that the cases which require higher brightness preservation and not improved well by HE, BBHE and DSIHE, have been accurately improved by RMSHE. MMBEBHE is the extension of BBHE method that provides maximal brightness preservation. Although these techniques can perform better contrast enhancement, these cause more infuriating side effects depending on the variation of gray level distribution in the histogram. DHE ensures uniformity in preserving image details and is free from any severe side effects. BPDHE can preserve the mean brightness better than BBHE, DSIHE, MMBEBHE, RMSHE, MBPHE, and DHE. MCBHE is simple and heuristic method for contrast enhancement in grayscale images and able to enhance the quality of images such that both global and local contrast is enhanced with minimum distortion in the image appearance. WMSHE achieves the best quality through qualitative visual inspection and quantitative accuracies of Peak Signal to Noise Ratio (PSNR) and Absolute Mean Brightness Error (AMBE) compared to other state of the art methods.

Cao et al.[2] proposed two novel algorithm detect the contrast enhancement involved manipulations in digital images. First, they dedicated to the detection of globalcontrast enhancement placed to the previously JPEGcompressed images, which are widespread in real applications. The positions of detected blockwise peak/gap bins are clustered for recognizing the contrast enhancement mappings placed on different source regions. The consistency between regional artifacts is checked for discovering the image forgeries and locating the composition boundary. Extensive experiments have verified the effectiveness and efficacy of the proposed techniques.

\section{CONCLUSION}

Image Enhancement is one of the important requirements in Digital Image Processing which is important in making an image useful for various applications which can be seen in the areas of Digital photography, Medicine, Geographic Information System, Industrial Inspection, Law Enforcement and many more Digital Image Applications. Image Enhancement is utilized to improve the quality of poor images. The focus of this paper is an attempt to improve the quality of digital images using Adaptive Histogram Equalization.

\section{REFERENCES}

[1] Agaian, S.S., Panetta, K. and Grigoryan, A.M. (2001), "Transform-based image enhancement algorithms with performance measure", IEEE Transactions on Image Procedureing, Vol.10, Issue: 3,pp. 367-382.

[2] Cao, Gang, Yao Zhao, Rongrong Ni, and Xuelong Li(2014). "Contrast enhancement based forensics in digital images."Information Forensics and Security, IEEE Transactions on 9, No. 3, pp.515-525.

[3] Chen, S.-D. and Ramli, A. R. (2003), "Minimum Mean Brightness Error Bi-Histogram Equalization in Contrast Enhancement”, IEEE Transactions on Consumer Electronics, vol. 49, no. 4, pp.1310-1319.

[4] Chen, S.-D. and Ramli, A. R. (2004), "Preserving brightness in histogram equalization based contrast enhancement techniques", Digital Signal Procedureing, vol. 14, pp. 413-428. 
[5] Garg, Rajesh, Mittal, Bhawna, and Garg, Sheetal (2011)," Histogram Equalization Techniques For Image Enhancement”, International Journal of Electronics \& Communication Technology (IJECT) Vol. 2, Issue 1.

[6] https://en.wikipedia.org/wiki/Adaptive_histogram_equalization

[7] Kaur, Manpreet, Kaur, Jasdeep and Kaur, Jappreet (2011), "Survey of Contrast Enhancement Techniques based on Histogram Equalization", (IJACSA) International Journal of Advanced Computer Science and Applications, Vol. 2, No. 7.

[8] Kim, Y. T. (1997), “Contrast Enhancement Using Brightness Preserving Bi-Histogram Equation”, IEEE Transactions on Consumer Electronics, vol. 43, no. 1, pp. 1-8.

[9] Radhi, Muna Abdul Hussain, Ban Sabah and Aurass Musa Omran Al-Hsniue(2014), "Enhancement Of The Captured Images Under Different Lighting Conditions Using Histogram Equalization Method", International Journal of Latest Research in Science and Technology, Volume 3, Issue 3.

[10] Sengee, N., Sengee, A. and Choi, H. K.(2010), "Image Contrast Enhancement using Bi-Histogram Equalization with Neighborhood Metrics", IEEE Transactions on Consumer Electronics, vol. 56, no. 4, pp. 2727-2734.

[11] Stark, J. Alex (2000), “Adaptive Image Contrast Enhancement Using Generalizations of Histogram Equalization" IEEE transactions on image procedureing, vol. 9, no. 5 .

[12] Tang, Jinshan, Peli, Eli, and Acton, Scott (2003), "Image Enhancement Using a Contrast Measure in the Compressed Domain", IEEE SIGNAL PROCEDUREING LETTERS, VOL. 10, NO. 10.

[13] Wang, Y., Chen, Q. and Zhang, B. (1999), "Image Enhancement Based on Equal Area Dualistic Sub-Image Histogram Equalization Method", IEEE Transactions on Consumer Electronics, vol. 45, no. 1, pp. 68-75.

[14] Zuo, C., Chen, Q. and Sui, X. (2012), "Range Constrained Bi-Histogram Equalization for Image Contrast Enhancement”. 